phosphate, die auf Basis der wasserlöslichen Phosphorsüure bezahlt werden.

Einige Fabrikanten behaupten, in dieser Hinsicht nachteiligen FinfluB beobachtet zu haben. Ich selbst hielt bis noch vor kurzer Zeit das System $\mathrm{Besk}$ ow allen andern bekannten Systemen für überlegen, bis ich zu Anfang dieses Jahres das System der Herren Ma l l ms te n \& Thor a ll (Aktiebolaget Svenska Konstgodnings och Svafvelsyre Fabrikerna. Malmö) kennen lernte. Dieses System ist meiner Ansioht nach erstaunlich einfach und vermeidet dabei jeden sohädlichen Einfluß auf den chemischen Proze $B$, so da $\beta$ ich keine Bedenken trage, es allen andern mir bekannten Systemen vorzuziehen. Erst nach längerer Vorbereitung, nach zweijährigem Betriebe und nachdem alle Konstruktionsteile gründlich ausprobiert waren, wurde die Erfindung patentiert und der Allgemeinheit zur wirtschaftlichen Verwertung angeboten.

Das Prinzip ist folgendes: Die genze Masse Superphosphat wird einer feststehenden Kratzvorrichtung zugeführt, in der Weise, daß die Masse nicht auf einem speziell dazu konstruierten Wagen herangezogen, sondern aus der etwas konisch gebauten, sonst in üblicher Weise gemauerten Kammer g e s c h o be n wird, um an der Stirnseite gleichzeitig abgekratzt zu werden. Man sollte denken, daß sich das Fortschieben einer so großen Masse wegen der dabei vorhandenen Bodenreibung schlecht ausführen läßt. In Wirklichkeit sieht man es mit erstaunlicher Leichtigkeit vor sich gehen. 3 PS. sind vollkommen genügend; ich habe mich persönlich davon überzeugt. Das Prinzip des Svenskasystems ist tatsächlich sehr originell. Das Kaiserl. Patentamt, welches sich zur Erteilung des Patentes neulich entschlossen hat, schreibt in seinen ,Gründen" $u$. m.:

„Es ist auch als eigenartig und überraschend anzusehen, da es von vornherein unwahrscheinlich erschien, daß sich das Verfahren ohne Beeinträchtigung der Eigenschaften des Superphosphates praktisch würde durchführen lassen. Die von den Einsprechenden erhobenen Einwände beruhen lediglich auf theoretischen Erwägungen und können den bestimmten Angaben der Patentsucherin gegenüber als stichhaltig nicht angesehen werden." -

Die Einrichtung ist folgende: Die Kammer wird in der üblichen Weise nach bestimmten Dimensionen gebaut oder umgebaut, bloß mit dem geringen Unterschied, daß sie von hinten nach vorn etwas konisch zuläuft.

In der üblichen Weise wird die Kammer gefült - alle Beeinflussungen der chemischen Reaktion bleiben also genau wie früher. Nach vollendetem Aufschluß wird die eiserne Türe geöffnet und das Schneckengetriebe in Gang gesetzt. Der guBeiserne Schieber 19 wird durch eine ebenso einfache wie sinnreiche Konstruktion von vier ganz in Öl laufenden Schnocken getrieben, an vier Stellen vorwärts gedrückt und schiebt den ganzen Ansatz Superphosphat langsam vorwärts. Dieses wird an der vertikalen Stirnseite durch Vorrichtung 24 kontinuierlich abgeschabt. Diese $\mathrm{Ab}$ schneidevorrichtung besteht aus zwei oder drei wagerechten Messern o. dgl., die an zwei Ketten befestigt sind, welche über Kettenräder auf beiden
Seiten der Öfnung laufen. Die Masse wird nur ganz wenig bearbeitet. Das abgeschnittene Superphosphat fällt auf Transportbahn 25 zur weiteren Beförderung.

Um zu verhindern, daß die Superphosphatmasse während ihrer Bewegung an den Seitenwänden der Kammer schleppt, sind die.Kammern nach der Öffnung zu ein wenig weiter. S o b a ld die Masse vorwärts geschoben wird, hört die Berührung mit den Wänden auf.

Die Vorzüge der mechanischen Kammerentleerung Svenska lassen sich folgenderweise zusammenfassen:

1. Jede Verschmierung des Superphosphates wird vermieden.

2. Die Kammerentleerung ist rein mechanisch. Ein Mann genügt zur Utberwachung.

3. Die Entleerungsmaschine befindet sich während des Mischens außerhalb der Kammer.

4. Die ganze Vorrichtung ist äußerst einfach und leicht zugänglich.

5. Die Löslichkeit des Superphosphats wird durch dieses System absolut nicht beeinflußt; und ein abnormales späteres Zurückgehen der Ware ist ausgeschlossen.

6. Jede Aufschließkammer kann für dieses Verfahren verwendet werden. Vorhandene Aufschließkammern wird man oftmals mit geringen Kosten nur etwas abzuändern brauchen.

7. Die Reparaturkosten sind sehr gering, etwa $1 \mathrm{M}$ per 1000 Tons Superphosphat.

Aus diesen und andern Gründen geht hervor, daß die mechanische Entleerung der Reaktionskammern gewaltige Fortschritte gemacht hat, und deshalb die Erwünschtheit oder Notwendigkeit eines kontinuierlichen Verfahrens zurückgedrängt ist. Mit Rücksicht aber auf die wirtschaftlichen Vorteile eines kontinuierlichen Verfahrens, welchem man, wie in jedem Betriebe, so auch bei der Superphosphatfabrikation nachstreben müßte, bleibe ich bei meiner Behauptung stehen, daß unsere Forschungen nach dieser $R$ ichtung $h$ in geleitet werden sollten.

[A. 108.]

\section{Die Verwendung der Elektrohängebahn im Betriebe chemischer Fabriken.}

Von Ingenieur Hubert Hermanss-Aachen.

(Eingeg. 19./6. 1910.)

Hänge- oder Schwebebahnen wurden früher ausschlieBlich in der Weise ausgeführt, daß an cinem hoch liegenden Gleise fahrende Transportgefäße an ein umlaufendes Drahtseil angekuppeit und von diesem mitgenommen wurden und so ihre Bewegung orhielten. Dieselben waren somit an eine zentrale Antriebsstation gebunden. Es ist eine allgemein bekannte Tatsache, welcher großen Beliebtheit sich solche Seilhängebahnen auch heute noch erfreuen und welche großen wirtschaftlichen Vorteile sich durch dieses Beförderungssystem bei der Bewegung von Massengütern erzielen lassen.

Nicht nur bei großen Fördermengen ist die Verwendung der Seilbahn unter Erzielung eines 
günstigen wirtschaftlichen Ergebnisses vorteilhaft, sondern auch für geringe Fördermengen, beispielsweise $5 \mathrm{t} / \mathrm{st}$, ist die Seilhängebahn noch mit Vorteil verwendbar, wenn gleich nicht verkannt werden
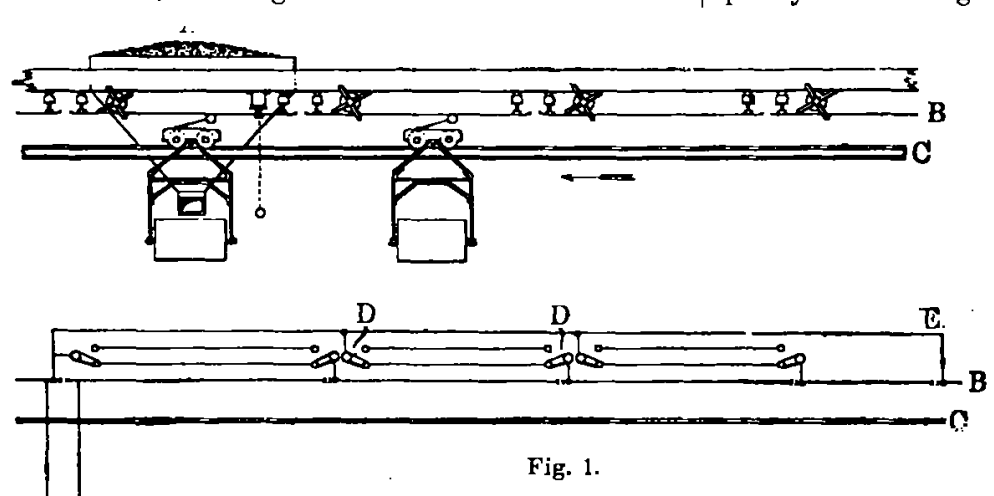

gekommene Bezeichnung von der bekannten Firma Adolf Bleichert \& Co. in Leipzig-Gohlis geprägt wurde, die sich um die Ausbildung dieses Transportsystems unvergängliche Verdienste erworben hat.

Die Elektrohängebahn ist demgernäB in ihrem Wesen dadurch gekennzeichnet, daß jedes einzelne Transportgefäß mit einem besonderen Antriebsmotor versehen ist, der dem Wagen die in Betracht kommenden Bewegungen und Arbeitsnotwendigkeiten vermittelt. Von der Seilhängebahn ist hierbei nur der hochliegende Schienenweg übernommen worden, welcher naturgemä $\beta$ noch mit einer elektrisohen Kraftlei-

kann, daß mit der zunehmenden Menge der zu befördernden Güter die Ausnutzung des Seiles sowohI als auch der Antriebsvorrichtung wächst, wadurch die größte Wirtschaftlíchkeit erzielt wird. Es könnte den Anschein haben, daß bei ungenügender Belastung des Seiles die Leerlaufverluste das wirtschaftliche Ergebnis erheblich za beeinflussen vermöchten, da indessen die Reibung der Tragrollen, die sehr leicht laufen, nur eine geringe ist, so fallen die Leerlaufsverluste nur unbedeutend ins Gewicht. Machen jedoch die Beschaffenheit des Geländes oder sonstige örtliche Verhältnisse viele Krümmungen und $A b$ lenkungen erforderlich, so stellt sich der Drabtseilbahnbetrieb verbietend teuer.

Es war somit das Bestreben wohl verständlich, sich einerseits die unverkennbaren Vorteile der Drahtseilhängebahn zu sichern, anderseits dieselbe jedoch in der Richtung zu verbessern, daß sich auch kleinere Transportmengen unter Erzielung einer ausreichenden Wirtschaftlichkeit mit derselben befördern ließen. Es war also vor allem nötig, eine Form für die Hängebahn zu finden, bei der sich die beim Seilbetrieb unvermeidlichen Leerlaufsverluste vermindern bzw. nach Möglichkeit vollatändig aufheben ließen. Dies ließ sich nur auf dem Wege erreichen, daB man die zentrale Antriebsstation fallen lie $\beta$ und jeden einzelnen Förderwagen mit

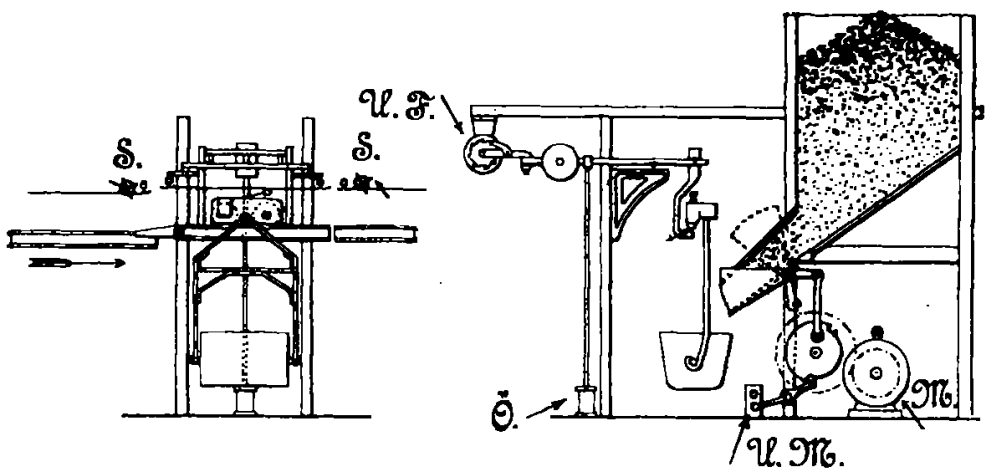

Fig. 2.

einem besonderen Antriebsmotor ausrüstete. So kam man auf die Hängebahn mit elektromotorischem Einzelantrieb, kurz Elektrohängebahn genannt, welche inzwischen allgemein in Aufnahme tung ausgerüstet werden muß, um den einzelnen Antriebsmotoren die zum Betriebe erforderliche Energie zuzuführen.

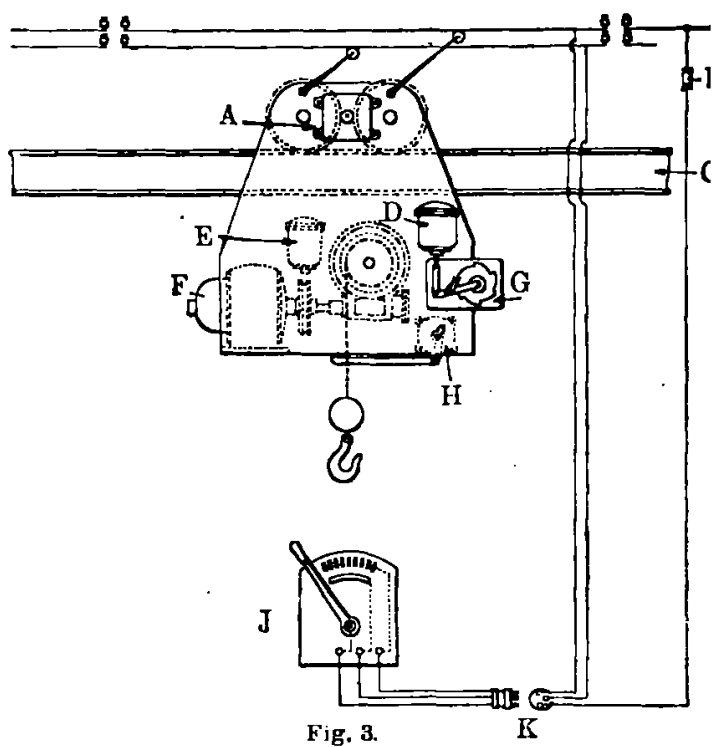

Wesentlich für den Begriff des elektroßutomobilen Hängebahnsystems ist indessen, daß die Steuerung der Arbeitsvorgänge auf automatischem Wege erfolgt, und da $B$ dieselben sich derartig selbsttätig sichern, daß Zusammenstöße und Kollisionen zwischen den einzelnen Fördergefäßen vermieden werden. Ihrem Wesen nach sind demgemäß Führerstandslaufkatzen, deren Steuerung durch einen mitfahrenden Maschinisten ausgeführt wird oder von Flur aus durch Steuerketten regulierte Motorlaufkatzen nicht als Elektrohängebahnen anzusprechen, da bei diesen automatischer Betrieb nicht in die Erscheinung tritt. Immerhin hängen dieselben hinsichtlich ihrer konstruktiven Einzelheiten und in ihrem Wesen mit diesen ursächlich zusammen, so 


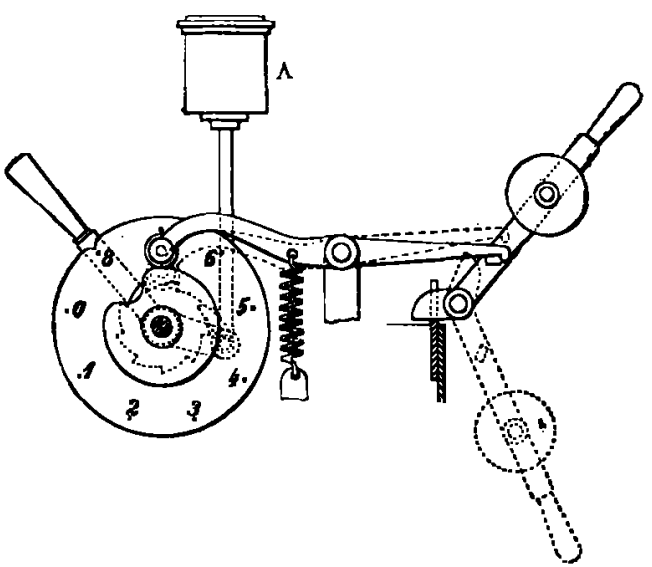

B
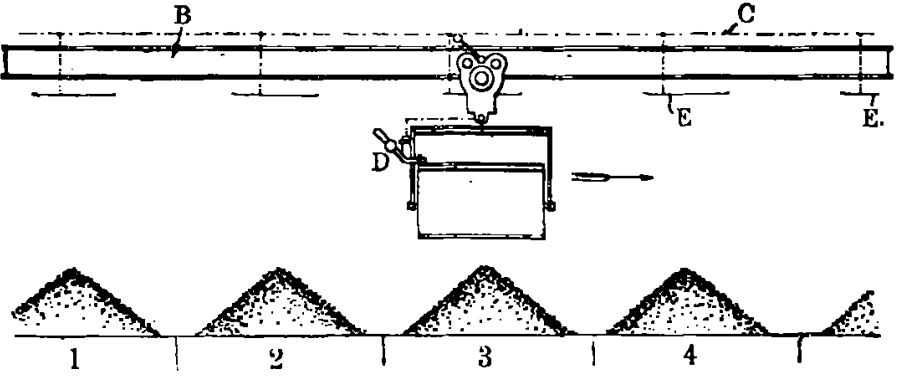

Fig. 4.

daß $B$ wohl angebracht sein dürfte, auch diese bei den nachfolgenden Erörterungen von einer kurzen Betrachtung nicht vollständig auszuschließen. Dies dürfte um so weniger angebracht sein, als dieselben je nach den im gegebenen Falle vorliegenden Verhältnissen dem automatischen Betriebe überlegen sein können. Dieselben habe ich sonach in den hier folgenden Ausführungen mit berücksichtigt.

Es dürfte nicht unwillkommen sein, auf die Vorzüge, welche die Elektrohängebahn gegenüber anderen Transportsystemen bietet, in Kürze hinzuweisen. Es seien folgende angeführt: .

1. Da es möglich ist, den Schienenweg für die Elektrohängebahn hoch zu verlegen, zumal da die Höho der Schienenverlegung bei der Ausrüstung der Wagen mit Windenvorrichtung praktisch keinen Beschränkungen unterliegt, so erfordert die Elektrohängebahn zu ihrem Betriebe keinen Bodenraum. Vielmahr kann dieser für Fabrikations- und sonstige Verkehrszwecke vollständig frei bleiben, und eine Störung der Fabrikations- und Erzeugungsvorgänge duroh den unproduktiven Transport ist ausgeschlossen. Die Linienführung der Bahn paßt sich sonach in weitestgehendem Maße den örtlichen Verhältnissen an, ein Umstand, der von besonderer Wichtigkeit für solche Betriebe ist, die mit beschränkten RaumverhäItnissen zu rechnen haben und darsuf bedacht sein müssen, den vorhandenen Raum ausschlieBlich der Fabrikation dienstbar zu machen.

2. Die Anpassung an die gegebenen örtlichen Verhältnisse ergibt sich auch aus dem Umstande, daB Kurven sowohl in horizontaler als auch in vertikaler Ebene durchfahren und beliebige Abzweigungen in die Bahn eingeschaltet werden können. In Verbindung mit der'Drahtseilbahn vermag die Elektrohängebahn als sog. Elektroseilbahn - auf die näheren Einzelheiten der Ele stroseilbahn wird weiter unten eingegangen werden - beliebige Steigungen "zu überwinden. Mittels eines in den Förderstrang eingeschalteten Aufzuges kann die Förderung sogar in senkrechter Richtung erfolgen, wobei allerdings der Aufzug selbst das eigentliche fördernde Element ist, während der Antriebsmotor des Wagens während des Hebens ausgeschaltet bleibt. Es ist somit wohl nicht zu viel gesagt, wenn man behauptet, daß die Elektrohängebahn das vielseitigste Transportmittel darstellt, eine ganzo Reihe von anderen Förderelementen $\mathrm{zu}$ ersetzen imstande ist und daher eine einheitliche und planmäßige Durchführung aller Transportnotwendigkeiten ermöglicht.

3. Ein sehr wesentlicher Vorzug, der im Elektrohängebahnprinzip begründet ist, besteht darin, da 8 sich dieselbe sowohl für kleine Fördermengen als auch für größte unter Erzielung ungefähr des gleichen wirtschaftlichen Nutzens eignet. Bei der Vergrößerung der zu befördernden Mengen läßt sich die Leistung durch Einstellen neuer Wagen in den Förderstrang jederzeit erhöhon, ohne daß eine Änderung der Einrichtungen erforderlich wäre, vorausgesetzt natürlich, daß der Querschnitt der Kupferleitung dem vermehrten Stromverbrauch angepaßt ist. Weiterhin kann aber auch bei der Vergrößerung der Fabrikanlagen die Bahnlinie ohne weiteres verlängert oder durch $\mathbf{A b}$ zweigungen erweitert werden.

4. Die Betriebs- und Bedienungskosten sind insofern billig, als der Kraftverbrauch durch geringe Leerlaufsverluste nicht hoch ist, und das erforderliche Bedienungspersonal auf ein Mindestmaß

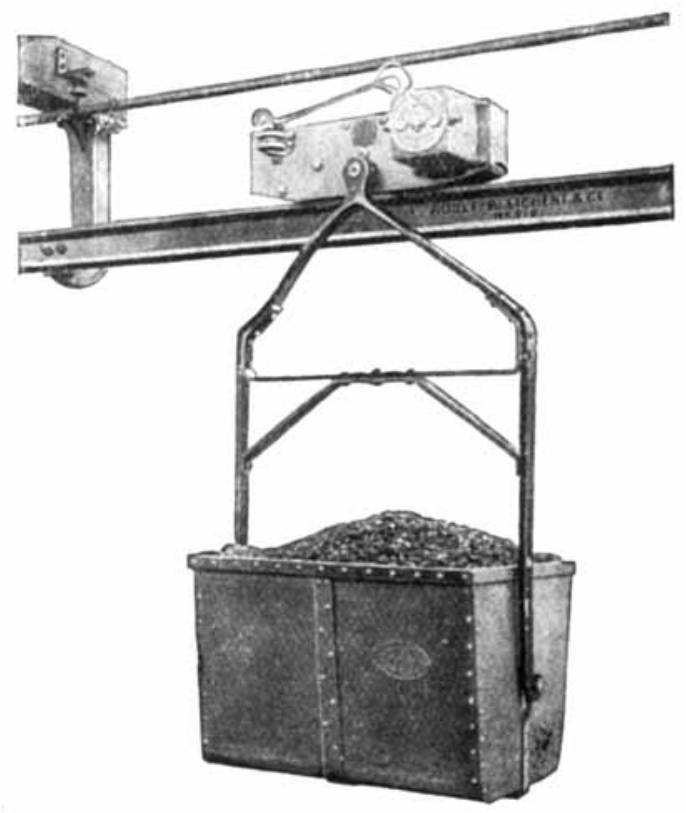

Fig. 5. 


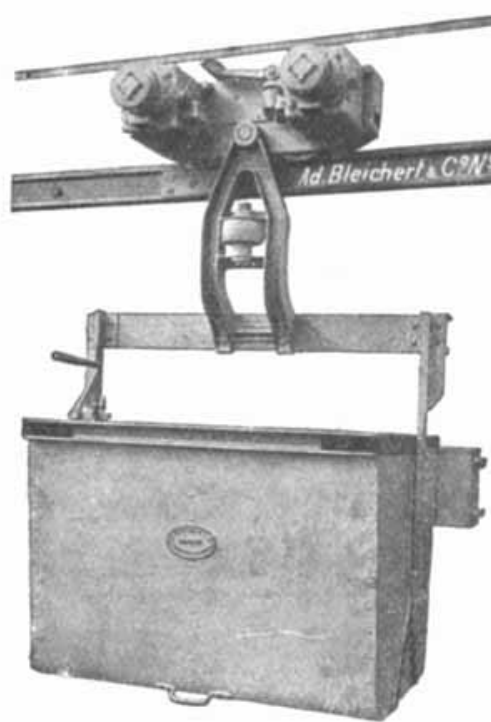

Fig. 6.

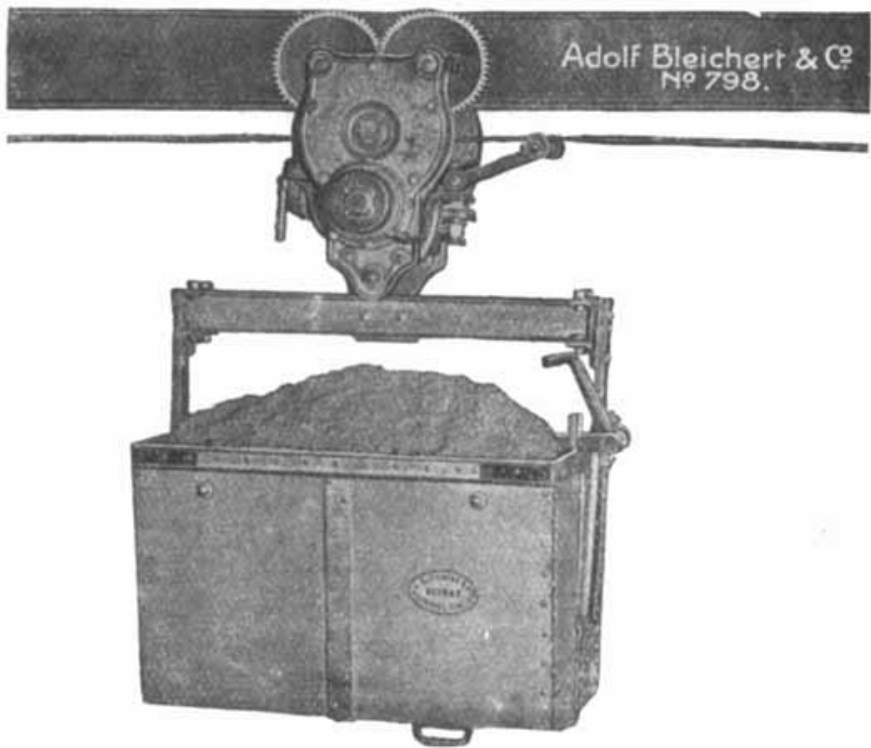

Fig. 7.

beschränkt zu werden vermag. Dies wird dadurch ermöglicht, daß Einrichtungen geschaffen worden sind, die einen sehr weitgehenden automatischen Betrieb und eine bemerkenswerte Unabhängigkeit vom bedienenden Arbeiter gewährleiston. Auf die in der Hauptsache in Betracht kommenden Einrichtungen, die darauf abzielen, einen selbsttätigen Betrieb der Elektrohängebahn zu ermöglichen, soll im nachstehenden in Kürze eingegangen werden.

Das Haupterfordernis beim Betriebe einer

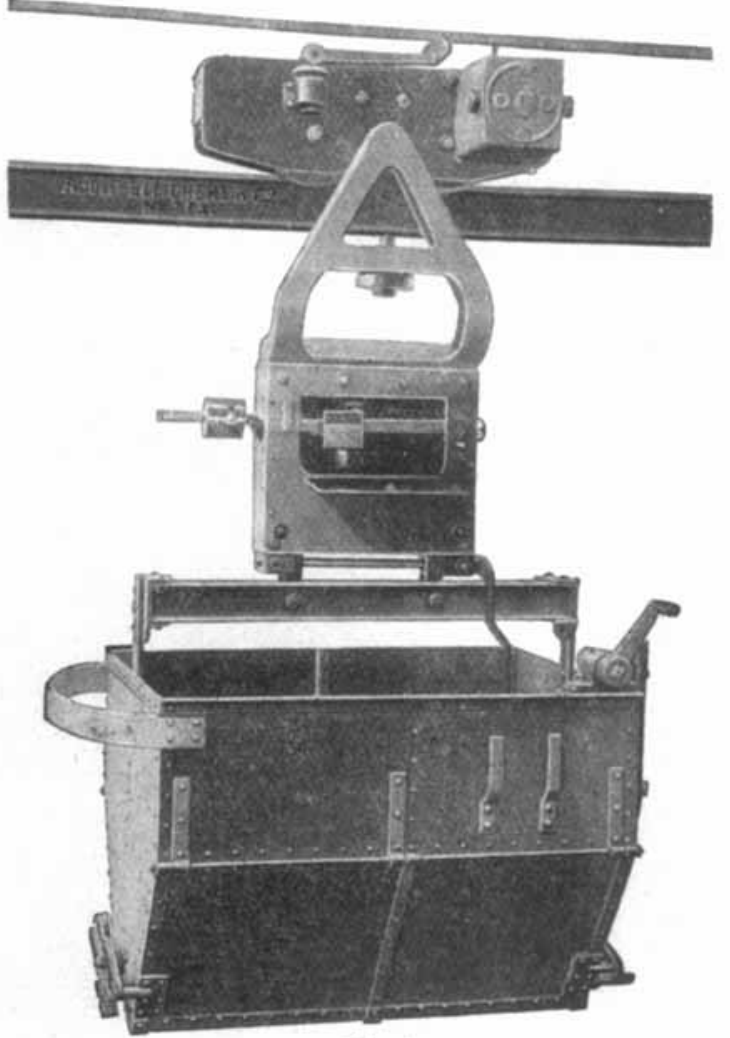

Fig. 8.

Elektrohängebahn, auf deren Fahrgleis mehrere Wagen im Ringbetrieb verkehren, ist naturgemäß, daß Zusammenstöße zwischen den einzelnen Fördergefäßen unmöglich gemacht werden. Diesen Zweck erreichten Adolf Bleichert \& Co.s deren Erzeugnisse und Konstruktionen im nachstehenden ausschließlich beschrieben werden sollen, durch eine selbsttätig wirkende Blockierungseinrichtung, die im Prinzip in der Weise arbeitet, daB die Fahrstrecke in mehrere Unterstrecken unterteilt ist, und durch den Wagen die gerade durchfahrene Strecke von der Speiseleitung abgeschaltet wird, so daß also dem folgenden Wagen die Stromzuführung abgeschnitten wird, und dieser sonach erst dann seins Fahrt wieder aufzunehmen vermag, wenn die Blockleitung wieder eingeschaltet wird. Schematisch ist eine Blockierungseinrichtung in Fig. 1 (S. 1401) dargostellt. Die in Betracht kommenden Arbeitsvorgänge vollziehen sich hier folgendermaßen. Aus dem Füll.

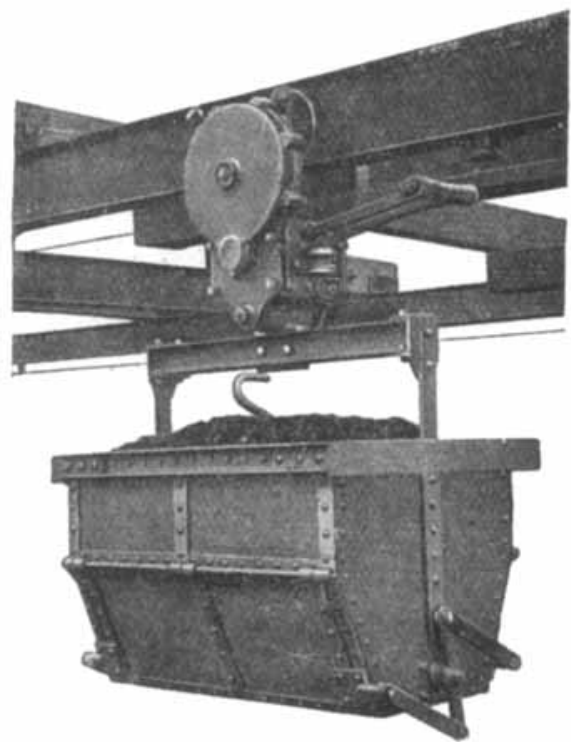

Fig. 8. 
rumpf A soll etwa der von der Fahrschiene $\mathrm{C}$ getragene und von der Stromleitung $B$ gespeiste Wagen beschickt werden. Auf der an dem Füllrumpf gelegenen Blockstrecke angelangt, macht der Wagen durch Drehen des Umschalters D die vorhergehende Strecke stromlos, während die erstere Strecke durch die Hilfsleitung E gespeist wird. Nach der Fiullung des Wagens wird der Hauptschalter F eingeschal. tet, worauf sich der Wagen in Bewegung setzt und nach Verlassen der Strecke die vorhergehende durch abermaliges Drehen eines Umschalters wieder unter Strom setzt, so daß der folgende Wagen wieder seine Fahrt fortsetzten kann. Weichen und Kreuzungen, die für Zusammenstöße in erster Linie in Be-

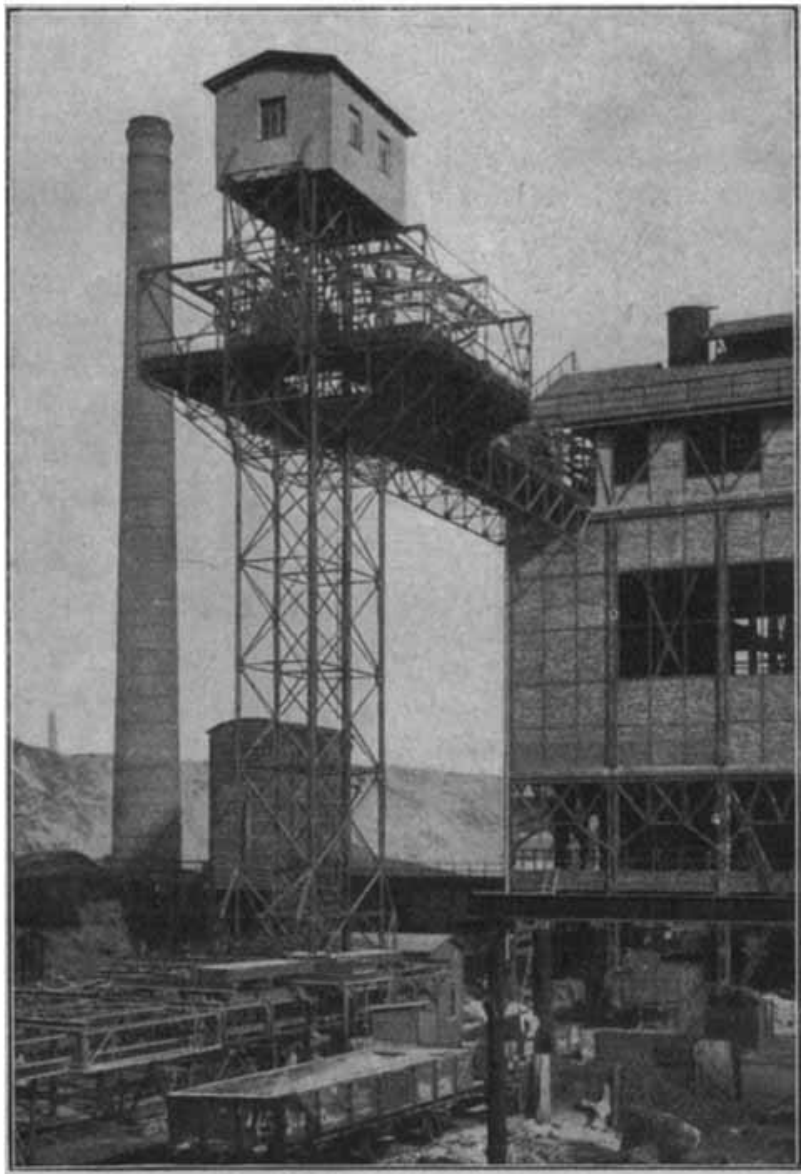

Fig. 10. tracht kommen, werden durch eine nach den gleichen Grundsätzen durchgebildete Einrichtung gesichert.

Bei der Füllung eines Wagens aus einem Füllrumpf ist demgemäB immer noch ein Arbeiter erforderlich, der das Füllen beaufsichtigt und den Wagen nach vollendeter Beschüttung wieder auf Fahrt schaltet. Auch diesen Arbeiter noch entbehrlich zu machen, die Füllung also auch selbsttätig durchzuführen, war nunmehr das naturgemäBe Bestreben der Ingenieure. Zur Erreichung dieses Zieles wurde die in Fig. 2 (S. 1401) abgebildete Vorrichtung konstruiert. Hier fährt der ankommende leere

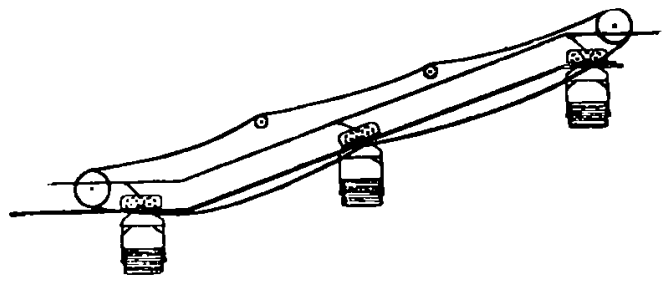

Wagen auf das als Wagebalken auggebildete Fahrschienenstïck auf und schaltet sich mittels desStreckensehalters S selbsttätig aus. Mittels des Motors M wird sodann die Verschlußklappe betätigt. Da sich mit dem zunehmonden Gewicht des sich füllenden Wagenkastens der Wagebalken senkt, so wird dio VerschluBkleppe des Füllrumpfes mittels des Umschalters UF und unter Zuhilfenahme des Ölkataraktes $\ddot{O}$ wieder angestellt, und der Motor des Wagens auf Fahrt geschaltet. Nach der Abfahrt des Wagens kehrt der Wagebalken wieder in seine ursprüngliche Lage zurück. Der Umschalter UM dient zum absatzweisen Aus- und Einschalten des Motors $M$ und der Fahrleitung über dem Wagebalken.

Unter Nr. 167893 wurde der Firma Adolf Bleichert \& Co. eine Einrichtung patentiert, die als automatische Fernschaltung für Windenwagen bezeichnet wird, und die dazu dient, bei großen Transportwegen, wo der Betrie $\dot{b}$ in sich regelmäBiglund periodisch wiederholenden Arbeitsvorgängen besteht, das Heben und Senken an den dafür vorgesehenen Punktenselbsttätig zu bewirken. Die Vorrichtung ist schematisch in Fig. 3 (S. 1401) abgebildet. Es ist hier eine besondere, von der Fahrleitung gespeiste Kontaktleitung vorgesehen. Die

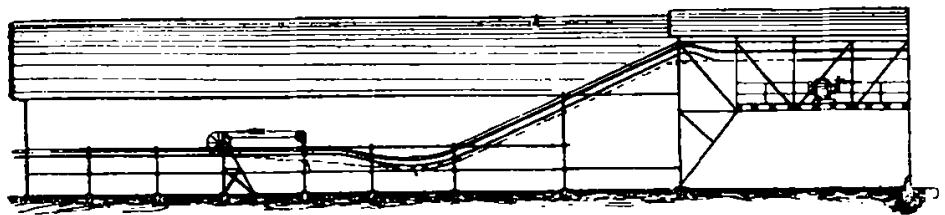

Fig. 11.

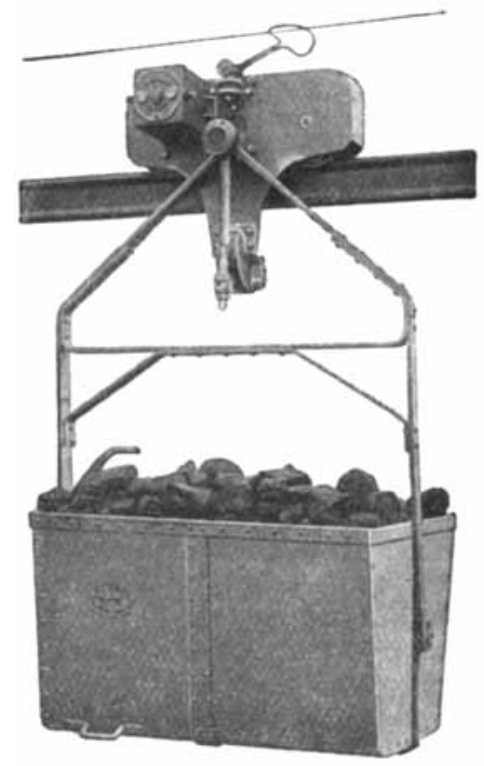

Fig. 12. 


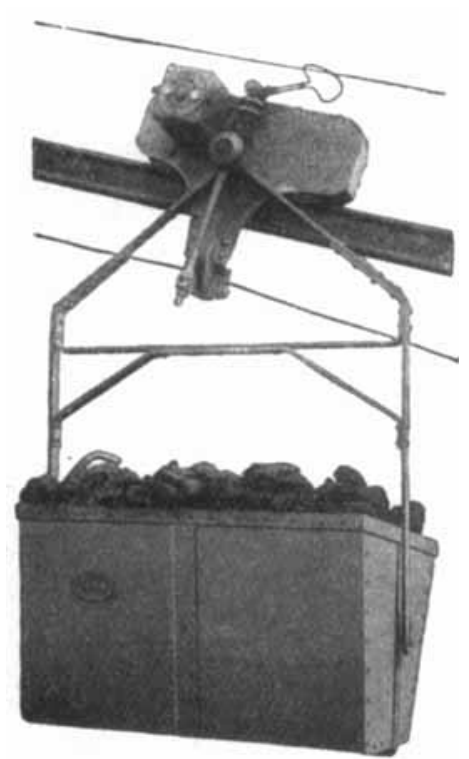

Fig. 13.

Hsuptleitung wird mittels des Anlassers $\mathbf{J}$ unter Strom gesetzt, welcher durch die Anschlußdose $K$ und die Sicherung $B$ nach dem Fahrmotor A flieBt. Der Schalt. magnet $D$ vermittelt die Verbin. dung mit der Schaltwalze G, die entsprechende Kontaktstücke besitzt, und von welcher dann die zugehörigen Schaltungen für die einzelnen Arbeitsvorgänge bewirkt werden. Der Strom braucht nur mittels des an irgend einem Punkte fest oder beweglich angebrachten Anlassers dem Hubmagneten D zugeführt zu werden. Der mit $\mathbf{E}$ bezeichnete Teil ist der Bremsmagnet für den Hub-

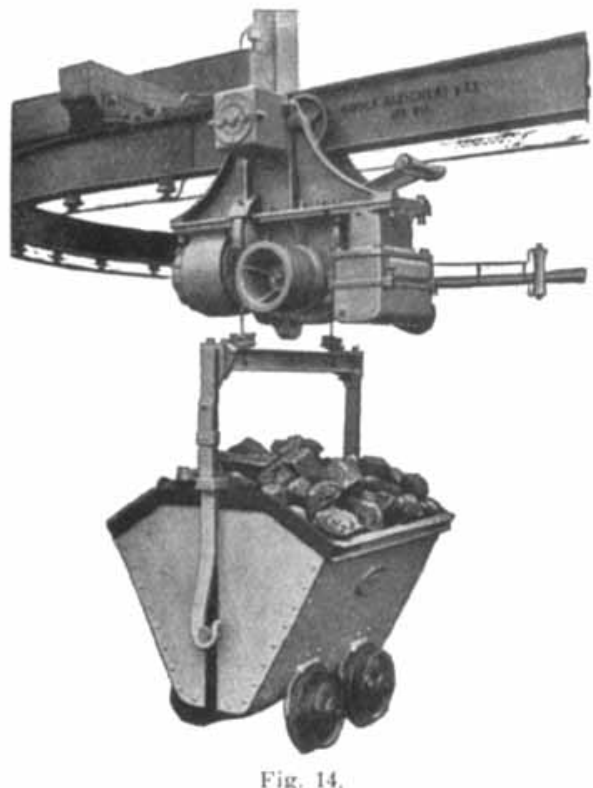
darstellt. motor, während $\mathrm{H}$ den Endausschalter

Die letzte Phase in der Entwicklung der Elektrohängebahn zum automatisch wirkenden Fördermittel ist die in Fig. 4 (S. 1402) schematisch wiedergegebene Einrichtung - unter Nr. 168512 patentiert - dio es gestattet, von einem festen Punkte aus die verschiedensten Arbeitsvorgänge, wie Heben und Senken, Kippen, Anhalten, Bedienung von Füllvorrich. tungen an verschiedenen Stellen der Gleis. strecke, Beeinflussen von Wägevorrichtungen, Betätigen der Blockierungsstrekken, außerdem noch bei verzweigten Bahnen das Umalegen von Weichen zu regulieren, wodurch auch der letzte Teil von Handarheit bei Massenverladungen beseitigt wird. Es wird hierbei an den jeweiligen Arbeitsstellen ein kurzes Stück der Stromleitung abgeschaltet, das dann von einem besonderen Stromabnehmer passiert wird, welcher einem Hubmag-

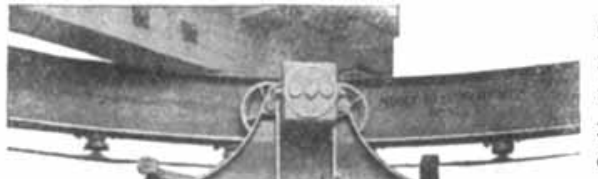

ein Wagen an verschiedenen Abwurfstellen gekippt werden. Die Einstellvorrichtung $D$ besteht aus einer Ziffernscheibe, auf welcher die einzelnen Kippstellen aufgezeichnet sind. Der mit Stellhebel versehene Zeiger wird nun auf die Entladestelle eingestellt. Der Zeigerhebel sitzt auf einer Welle mit Schaltrad, das so viel Zähne besitzt als dieScheibe Zeichen aufweist. Das Gesperre besteht aus einer Kurvenscheibe mit federbelast etem Hebel und einer

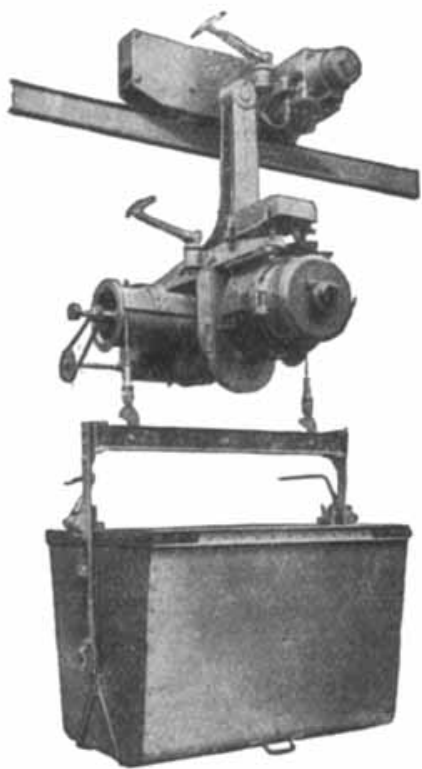

Fig. 17. neten oder dem in Frage kommenden Motor Strom zufïhrt. Ein hierbei in Tä. tigkeit tretendes Schalt. werk, mit dessen Schaltrad eine Kurven- oder Stufenscheibe verbunden ist, wird bei jedem StromstoB um einen Zahn fortgeschaltet. Durch Einschnappen einer Klinke in eine der Aussparungen tritt eine Ent- oder Verriegelung ein, so da $B$ der gewünschte Arbeitsvor. gang automatisch eingeleitet wird.

Bei dem in Fig. 4 wie. dergegebenen Beispiel soll

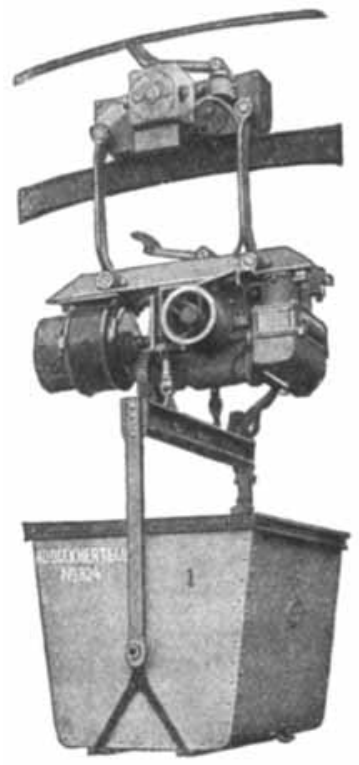

Fig. 16

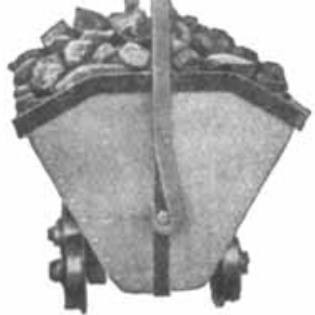

Fig. 15. 

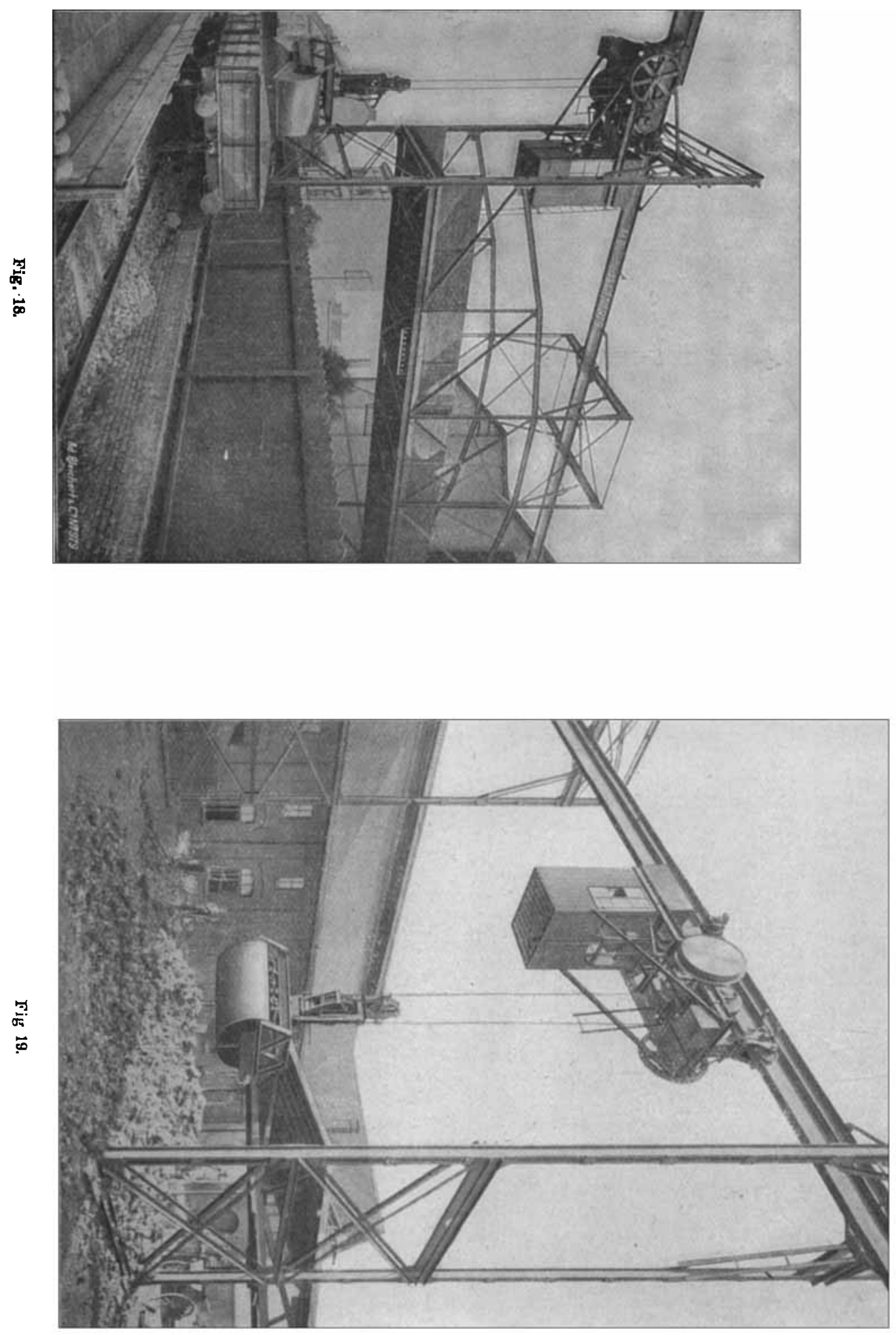
Sperrklinke. Der Hebel wird nun, wie bereits erwähnt, auf die Stelle eingestellt, an welcher die Entleerung des Wagens erfolgen soll. Durch den Magneten wird das Gesperre an den Punkten, wo nicht gekippt wird, um einen Zahn vorgeschoben, bis der Einstellkegel sich an der Kippstelle auf $O$ zurückgedreht hat. Jetzt fällt der Feststellhebel in den Kurvenscheibenausschnitt, so daß der Wagenkasten freigegeben und gekippt wird.

Nachdem nunmehr die Elektrohängebahnen hinsichtlich ihrer automatischen Arbeitsweise behandelt worden sind, dürfte es wohl angebracht und von Interesse sein, versehiedene Typen von Elektro-
Einen Elektrohängebahnwagen einfacher Form zeigt Fig. 5 (S. 1402). Der Wagen bewegt sich auf einer Kopfschiene und ist mit nur einem Fahrmotor ausgestattet, welchem der Strom durch eine oberhalb der Schiene ausgespannte Fahrleitung zugeführt wird. Die Ausbildung des Gehänges entspricht im allgemeinen derjenigen, wie sie bei Seilhängebahnwagen üblich ist, wie auch die Ausführung des Kastens, welcher sich durch Kippen entleert, von der Seilhängebahn übernommen ist.

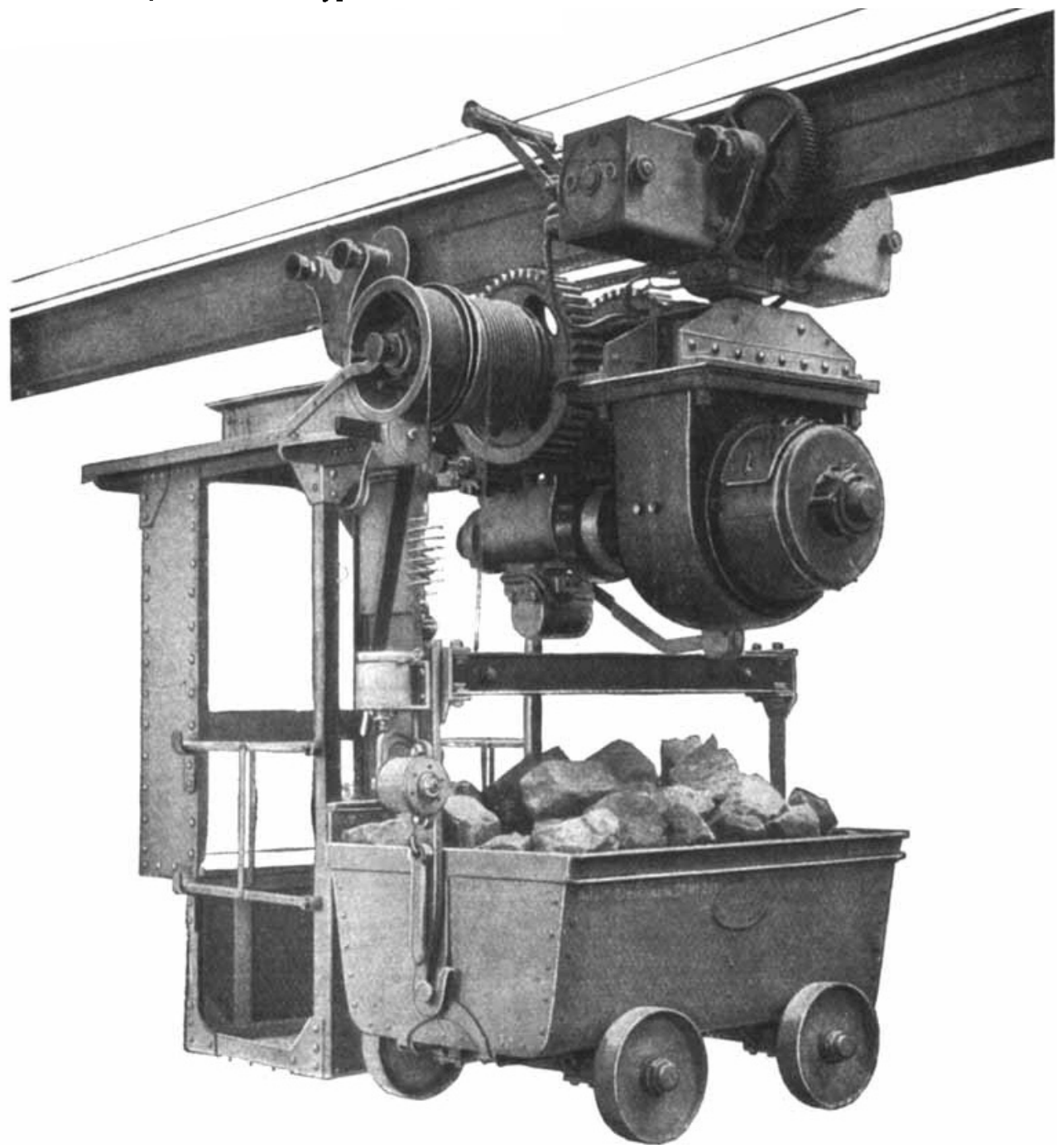

Fig. 20.

hängebahnwagen im Bilde vorzuführen und bezüglich ihrer besonderen konstruktiven Eigentümlichkeiten zu besprechen. Ganz allgemein möge dazu erwähnt werden, daß man zu unterscheiden hat zwischen einfachen Elektrohängebahnwagen, also solchen, welche sich lediglich auf horizontaler Strecke zu bewegen vermögen; ferner solchen, die mittels einer besonderen Einrichtung auch Steigungen überwinden können, die sog. Elektroseilbahn; endlich solchen, die außer dem Fahrwerk mit einer Windenvorrichtung ausgerüstet sind.
In ähnlicher Weise ist der in Fig. 6 (S. 1403) veranschaulichte Wagen ausgebildet. Jedoch unterscheidet sich derselbe von dem in Hig. 5 wiedergegebenen dadurch, daß das Fahrwerk zwei Motoren besitzt, welche den Wagen in den Stand setzen, auch Steigungen zu befahren. Das Gehänge ist noch mit einer Leitrolle versehen, um in starken Kurven der Zentrifugalkraft entgegenzuwirken.

In Fig. 7 (S. 1403) ist ein an dem unteren Flansch einer I-Eisenschiene fahrender Wagen abgebildet. Aus der veränderten Schienenform ergab sich eine ab- 
weichende Durchbildung des Fahrwerkes, das nur einen Antriebsmotor besitzt. Der die Stromzuführung vermittelnde Fahrdraht ist unterhalb der Fahrschiene ausgespannt.

Der in Fig. 8 (S. 1403) wiedergegebene Elektrohängebahnwagen unterscheidet sich von dem in Fig. 5 abgebildeten nur dadurch, daß in das Gehänge eine Wiegeeinrichtung eingebaut ist, die es gestattet, das Gewicht der jeweils geförderten Materialien festzustellen. Die Entleerung des Kastens erfolgt durch Offnen der Bodenklappen.

Fig. 9 (S. 1403) zeigt ebenfalls einen mit Bodenentleerung ausgestatteten Elektrohängebahnwagen. Das Fahrwerk desselben besitzt nur einen Motor, so daß der Wagen nur auf horizontalen Strecken oder normalen Steigungen zur Verwendung gelangen kann. Sind auf der Fahrstrecke starke Steigungen vorhan-

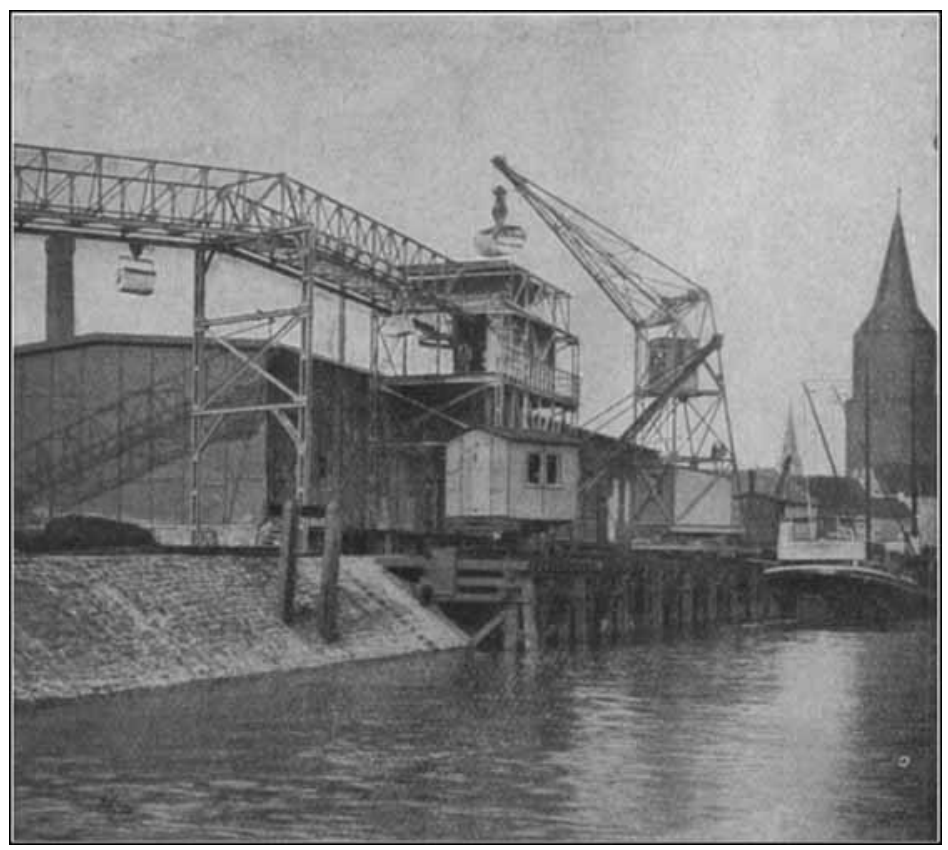

Fig. 21.

den, die das Fördergut zu überwinden hat, wie dies z. B. bei der Begichtung von Kuppel- oder Hochöfen der Fall ist, so werden die Bahnen duroh einen zwischengeschalteten Aufzug miteinander verbunden. Ein Aufzug dieser Art ist in Fig. 10 (S. 1404) zur Darstellung gebracht.

Eine andere Möglichkeit der Uberwindung von starken Steigungen bietet die Elektroseilbahn, die in sohematischer Wiedergabe in Fig. 11 (S. 1404) abgebildet ist. Man hat hier zu einer Kombination zwischen zwangläufigem und kraftschlüssigem Betriebegegriffen, indem man Hub- und Reibungsarbeit vollständig voneinander trennte, wobei beide Arbeiten von besonderen Maschinenelementen geleistet werden. Auf dem horizontal liegenden Streckenteil geschieht der Betrieb rein elektrisch mit Hilfe der Motoren. Dagegen ist die Steigungsstrecke mit einem umlaufenden Zugseil ausgerüstet, an welches sich die Wagen durch einen Mitnehmer oder eine Zugseilklemme selbsttätig ankuppeln. Die Bewegung des Ankuppelns wird gleichzeitig dazu be- nutzt, den Motor des Fahrwerkes automatisch auszuschalten. Nunmehr wird der Wagen mittels des Zugseiles die Steigungsstrecke hinaufgeschleppt, worauf sich derselbe am höchsten Punkte der Steigung wieder selbsttätig abkuppelt, indem gleichzeitig der Fahrwerksmotor wieder automatisch eingeschaltet wird und sioh auf der horizontalen Strecke der Wagen wieder mit Hilfe des Motors weiter bewegt. Fig. 12 (S. 1404) zeigt einen Elektroseilbahnwagen auf der horizontalen Strecke, während der in Fig. 13 (S. 1405) abgebildețe Wagen auf der ansteigenden Strecke fahrend dargestellt ist.

Ein weiteres und verhältnismäßig einfaches und betriebssicheres Mittel, starke Steigungen und Höhenunterschiede zu überwinden, ist dem Ingenieur auch durch den elektrischen Windenwaigen an Hand gegeben, der in Fig. 14 (S. 1405) zur Darstellung gebracht ist. Man kann hier den Schienenweg so hoch verlegen, $\mathrm{daB}$ ansteigende Streckenteile in der Bahn nicht mehr erforderlich sind. Der Wagenkasten wird an der tiefer gelegenen Stelle abgelassen, darauf beladen und wieder hochgezogen. An der höher gelegenen Stelle wird sodann zum Zwecke der Entladung der Kasten ebenfalls abgelassen. Natürlich können sich die genannten Arbeitsvorgänge auch in umgekehrter Reihenfolge abspielen. Der Betrieb kann sich hierbei, wie ich bereits oben gezeigt habe, vollkommen automatisch abwickeln. Die Senkbewegung der Winde kann auch auf eine bestimmte Hubhöhe eingestellt werden mittels einer Vorrichtung, die an dem in Fig. 15 (S. 1405) abgebildeten Windenwagen sichtbar ist. Dieselbe besitzt einen Endausschalter, der aus einer am Windenwagen gelagerten Spindel mit auf dieser sich verschiebendem Stein besteht, mittels dessen die Schaltwalze betätigt wird. Die Spindel wird durch ein Kettengetriebe angetrieben, welches die Welle der Windentrommel mittels eines aufgesetzten kleinen Zahnrades mit der Spindel verbindet. Durch entsprechende Einstellung der Spindel gegenüber dem Stein läßt sich praktisch ganz genau die Endstellung des zu senkenden Wagens sowohl in seinem höchsten als auch in seinem niedrigsten Punkte regulieren.

Einen Windenwagen ähnlicher Bauart läßt Fig. 16 (S. 1405) erkennen. Da jedoch dieser Wagen auf einer Kopfschiene fährt, so mußte das Fahrwerk, welches oberhalb der Fahrschiene angeordnet ist, von dem Hubwerk getrennt ausgeführt werden. Der in Fig. 17 (S. 1405) wiedergegebene Wagen weioht von dem in Fig. 16 veranschaulichten insofern $a b$, als die Lage der Hubtrommeln, welche gewöhnlich zur Erzielung einer guten Ausbalanzierung in der Richtung der Schienenlinie liegen, eine von der Norm abweichende ist, indem dieselben quergestellt sind. Im übrigen entspricht dieser Wagen dom in Fig. 16 abgebildeten in allen Teilen. 
Soll die Beladung der Elektrohängebahnwagen von einem zu ebener Erde gelegenen Lager aus erfolgen, so muß, wenn man Handverladung vermeiden will,-der Wagenkasten als Selbstgreifer ausgebildet werden. Die in Fig. 18 (S. 1406) abgebildete Anlage dient zur Entladung von Kohlen aus Fisen- erforderlich ist, so da B dieselben in weitestgehendem Maße geschont werden. In derselben Weise vollzieht sich bei dieser Anlage, die auf dem Gaswerk der Stadt Neuß in Betrieb ist, die Koksaufnahme vom Lagerplatz, die in Fig. 19 (S. 1406) wiedergege ben ist. Der Koks wird mittels des Selbstgreifers vom Lage

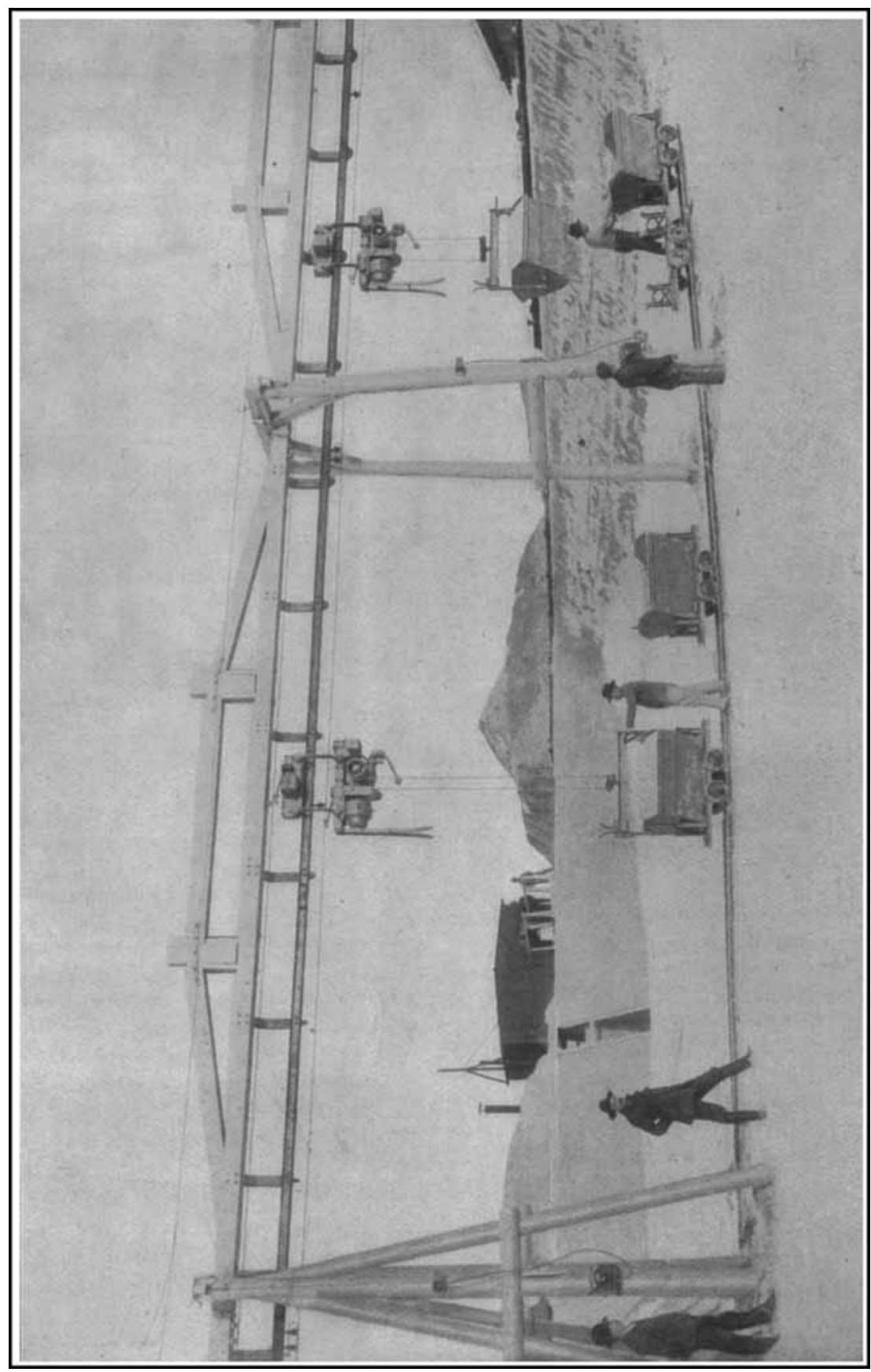

bahnwagen und zum Transport derselben nach der Verbrauchsstelle. Es liegt auf der Hand, daß sich bei diesem Verladesystem außerordentliche Ersparnisse insofern erzielen lassen, als nicht nur die Beladung der Fördergefäße auf mechanischem Wege erfolgt und keiner Hilfe von Menschenhand bedarf, sondern auch kein Zwischenumschlagen der Materialien aufgenommen und den Eisenbahnwagen zugeführt. Es versteht sich von selbst, $\mathrm{da} B$ man hier zu einem Teile auf die Vorteile des automatischen Betriebes verzichten mußte, da sowohl die Ausführung des Förderkastens als Selbstgreifer als auch die zurückzulegenden verschiedenen Transportwege es erforderlich machten, daß zur Steuerung des Wagens der 


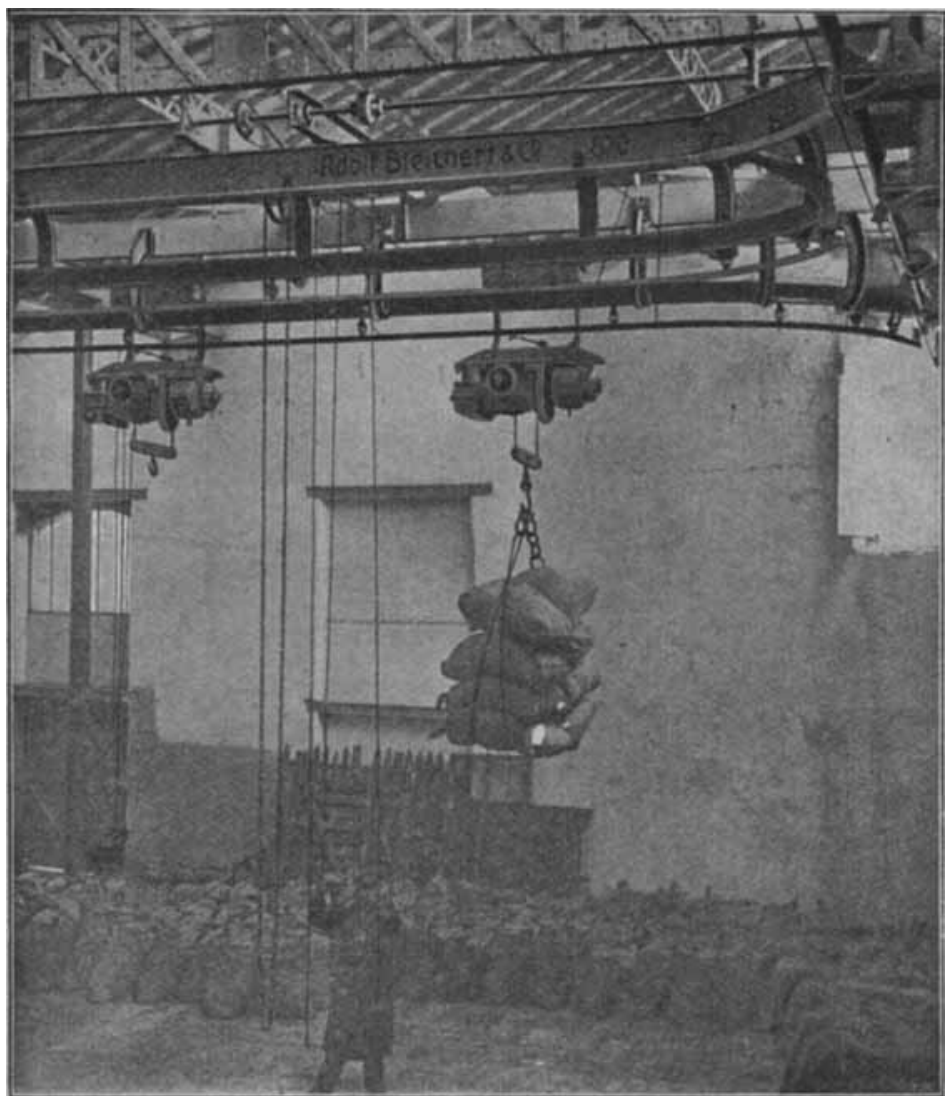

Fig. 23.

Auslauföffnung des Trichters vorbeigeleiteten Elektrohängebahnwagen, welche dasselbe sodann nach dem Lagexschuppen verbringen und hier auskippen. Nach der Entleerung kehren die Wagenkasten wieder selbsttätig zur Beladestelle zurück, um eine neue Ladung in Empfang zu nehmen.

Ein Beispiel einer automatisch wirkenden Elektrowindenbahn ist in Fig. 22 (S. 1409) veranschaulicht. Diese Anlage dient zum Transport von Kalkschlamm. Das Material wird in den auf Fahrgestellen stehenden Muldenkippwagen herangefahren und darauf letztere in das Gehänge des Elektrowindenwagens eingehängt und zur Verbrauchsstelle befördert. Nach der Entleerung kehren die Wagen wieder zurück und werden auf die Fahrgestelle abgesetzt, um wieder gegen gefüllte Kasten ausgetauscht $\mathrm{zu}$ werden.

Die in Fig. 23 abgebildete Anlage einer französischen Zuckerraffinerie dient zum Aufstapeln und Lagern der Säcke im Magazin und ihrem Weitertransport in die Sie-

zu bewegenden Last ein Steuermann beigegeben wird, der stets mitfährt.

Eine weitere elektrisch betriebene Windenlaufkatze zeigt noch Fig. 20 (S. 1407). In der Regel gelangen Windenlaufkatzen dieser Art dort zur Anwendung, wo an einer Reihe von Arbeitsstellen gehoben und gesenkt werden soll, die von einem festen Punkte aus nicht gut ïbersehen werden können. Fährt der Steuermann mit dem Wagen, so hat er die zu senkende Last stets unmittelbar vor Augen. Die Entleerung des Wagens kann sodann durch den Wagenführer an jeder beliebigen Stelle und in jeder Höhe vorgenommen werden.

Nach dieser, wenn nicht lückenlosen, so doch immerhin ziemlich vollständigen Zusammenstellung der Typen von Elektrohängebahnwagen, wie sie für die Bewältigung von Transporten in chemischen Betrieben in Frage kommen, möge es mir getattet sein, noch einige ausgeführte und in chemischen Fabriken in Betrieb befindliche Anlagen vorzuführen und hinsichtlich ihrer besonderen Eigentümlichkeiten und ihres Betriebəz in Kürze zu behandeln.

In Fig. 21 (S. 1408) sind die Transporteinrichtungen einer Düngerfabrik zur Darstellung gebracht. Es handelt sich hier darum, den in Schiffen ankommenden Rohphosphat zu entladen und nach: den Lagerschuppen zu transportieren. Zu diesem Zwecke ist am Ufer ein elektrisch betriebener fahrbarer Drehkran aufgestellt, welcher mittels eines angehängten Selbstgreifers das Material aus den Schiffen hebt und in einen hochliegenden Fülltrichter"entladet. Aus diesem fällt das Transportgut in die on der derei. Die Bahn bildet ein geschlossenes Rechteck, dessen Längsseiten an den Wänden des Speicherraumes liegen, während die eine Querschiene außerhalb des Gebïudes verlegt ist, so daß hier die Säcke von den Fuhrwerken abgenommen und gehoben werden können. Die zweite Querschiene befindet sich in der Siederei. Im Speicher endlich ist eine verfahrbare Brücke vorgesehen, deren Schienen sich mit aufklappbaren Zungen an die Längsstränge anschlieBen. Auf diese Weise entstehen zwei in sich ge-

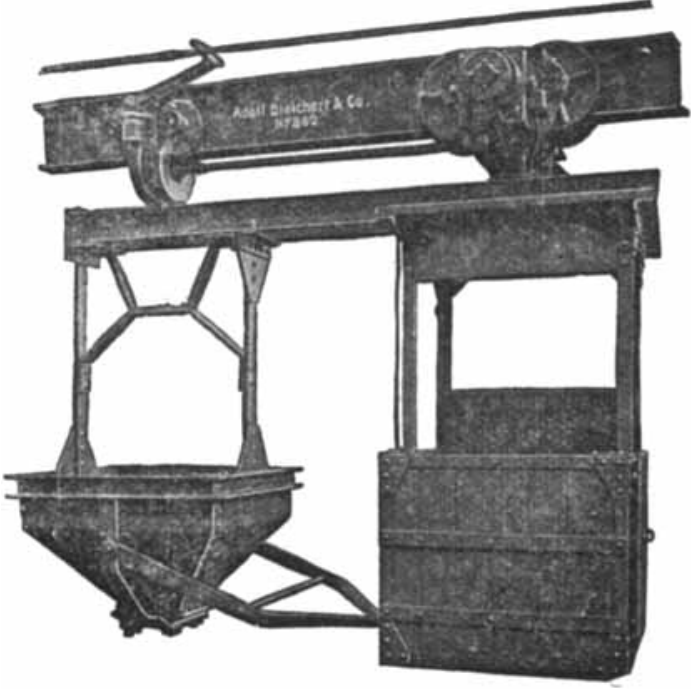

Fig. 24. 
schlossene Ringbahnen, auf denen die Wagen nach Bedarf zum Lagern der Säcke oder zum Uberladen nach den Fabrikationsräumen benutzt werden.

Bei der in Fig. 24 gezeigten Einrichtung, die zum Transport von Schwefelkies bestimmt ist, war die Forderung gestellt, daß der Förderkübel an verschiedenen Stellen genau über den Aufschütttrichtern der Öfen entleert werden sollte. Diese Aufgabe ließ sich in der einfachsten Weise derartig lösen, daß dem Wagen ein Steuermann mitgegeben wurde, welcher mittels eines Kontrollers den Wagen steuert und mittels eines Hebelgestänges die Öffnung der Bodenklappe des Kübels reguliert. Der Steuermann besorgt auch die Beladung des Kübels selbst, indem er von seiner Kabine aus den Hebel des Füllrumpfverschlusses dreht, so daß weitere Bedienung für die Bahn nicht erforderlich ist.

Es versteht sich von selbst, daß eine solche Anlage nur dort mit Vorteil angewendet werden kann and wirtschaftlich zu arbeiten vermag, wo die zu be- wältigenden Fördermengen nur einem Wagen Arbeit geben. Die Anlage wïrde dagegen dort unpraktisoh sein, wo der Transport mittels mehrerer Wagen erfolgte, da in diesem Falle die Lohnausgaben so hooh würden, daß das wirtschaftliche Ergebnis nicht unbeträchtlich herabgesetzt werden würde.

Wo die hier angedeuteten Verbältnisse vorliegen, kann aber sehr wohl mit Hilfe von Krananlagen für die vertikale Förderung der Betrieb durchgeführt werden. In diesem Falle wird die Horizontalförderung vom Drahtseilbahn- oder Elektrohängebahnwagen übernommen. Die Firma Bleichert \& Co. hat eine ganze Reihe derartiger kombinierter Anlagen ausgeführt, wobei die Beladung der Schwebebahnwagen entweder durch Krane erfolgt oder aber so durchgeführt wird, daß der Kran den Schwebebahnwagen von den Hängebahnschienen abnimmt, zur Beladestelle abläßt und nach der Beladung wieder auf die Schienen aufsetzt.

[A. 112.]

\section{Wirtschaftlich-gewerblicher Teil.}

\section{Jahresberichte der Industrie und des Handels.}

Cuba, Am A $\mathrm{B}$ enh ande $1 \mathrm{Cu}$ bas i. J. 1909 (1908), der sich in der Einfuhr auf 91026781 (85 218 391), in der Ausfuhr auf 124711039 (94 603 324) Doll. bewertete, war Deuts ch l a n d folgendermaßen beteiligt: Einfuhr 6562411 (7 172 358), Ausfuhr 4053960 (4 711 164) Doll.

$$
\text { - l. [K. 799.] }
$$

Dominlksisehe Republik. Der A u $B$ o h a $n$ del der Dominikanischen Republik i. J. 1909 bewertete sich auf 12539603 Doll. (1 624659 Doll. weniger als 1908); der Rückgang ist hauptsächlich auf die verminderte Kakaoausfuhr zurückzuführen und verteilte sich mit 1282797 Dollar auf die Ausfuhr und 341862 Doll. auf die Einfuhr. Die Einfuhr erreichte einen Wert von 4425913 , die Ausfuhr von 8113690 Doll. Nach den Vereinigten Staaten von Amerika wurden 58\% der Ausfuhr versandt im Werte von 4709354 Doll., während $54 \%$ der Einfuhr im Werte von 2374025 Dollar von dort kamen. Das zweite Hauptland für den Dominikanischen Handel war D e u t s c h la $\mathrm{nd}$, aber der Handelsverkehr zwischen beiden Ländern erlitt eine Einbuße in Höhe von 1994416 Dollar; die Ausfuhr nach Deutschland fiel gegenüber 1908 um rund 2 Mill. Doll., die von Kakao allein um 2154024 Doll. Dagegen vermochte Deutschland etwas mehr als im Vorjahre in San Domingo abzusetzen, nämlich für 911976 Doll. (gegenüber 868230 Doll. i. V.), was von keinem anderen Haupthandelslande gesagt werden kann. - Die A u s u hr von Zucker betrug $69483 \mathrm{t}$ i. W. von 3304931 Doll., Kakao 14586 t i. W. von 2759191 Doll., Wachs 477599 Pfd. i. W. von 123769 Doll., Holz einschließlich Mahagoni und Guajak 124377 Doll. - E in $f$ u h r : Fisen und Stahl 609416 Doll., davon aus den Vereinigten Staaten 385 221, Großbritannien 141 381, Deutschland 62885 (reichlich um $50 \%$ mehr als wpon
226065 Doll., Chemikalien, Drogen und Farben 88307 Doll., Zucker und Konfekt 79055 Doll., Flaschenbier 68702 Doll. (aus Deutschland allein 57 035), Seife 68669 Doll. (meist aus den Vereinigten Stasten), Fett und Abfallett zur Seifenfabrikation 58569 Doll. (aus den Vereinigten Starten und Deutschland), Papier und Papierwaren 52846 Doll. (die Hälfte aus Deutschland). (Nach Bulletin of the International Bureau of the American Republics.) -l. [K. 852.]

Australien. Der $A$ u Benhandel des A ustralischon Bundes i. J. 1909 stellte sich folgendermaßen: Einfuhr 51117000 (1908: 49799000 ), Ausfuhr 62917000 (62 119 000) Pfd. Sterl, der Wert der ein- und ausgeführten Edelmetalle und Mänzen ist in diesen Zahlen mit enthalten. Von einigen der wichtigsten Warengattungen seien folgende Werte in $1000 \mathrm{Pfd}$. Sterl. angegeben. E i n $\mathrm{u}$ h r: Bier und Ale 377 (413), Drogen und Chemikalien 958 (1117), Glas und Glaswaren 372 (372), Gold, ungemünzt 1007 (951), Kautschukwaren 485 (412), Eisen und Stahl in Stangen, Stäben und Trägern 992 (990), verzinkte Eisenplatten und -bleche 1442 (1128), Weißbleche 285 (285), Maschinen (ausgenommen landwirtschaftliche) und $\mathrm{Ma}$ schinenteile 2881 (2771), Metallwaren 3085 (3392), Kerosen 630 (484), Farben, Farbstoffe und Firnisse 414 (430), Papier 1146 (1367), Spirituosen 996 (1029). - A u s f u h r : Kohle 838 (1349), Kupfer in Ingots und Matte 1907 (2155), Gold, ungemünzt 3601 (3031), Goldmünzen 4320 (9820), Bleimulde und Bleimatte 810 (1346), Erze, außer Golderz 764 (928), Silber in Barren und Matte 656 (1068), Talg 1230 (785), Zinn in Ingots 754 (809). (Nach Account relating to the Trade and Commerce of certain Foreign Countries and British Possessions.) -l. [K. 813.]

Ceylon. tber die Beteiligung Deutschlands am Einfuhrhandel Coylons i. J. 1909 entnehmen wir einem Berichte des Kaiserl.

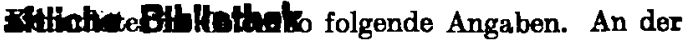

